\title{
AGREEMENTS AND DISAGREEMENTS BETWEEN THEORIES OF RIGID EARTH NUTATION
}

\section{J. SOUCHAY}

Observatoire de Paris

Paris, France

\begin{abstract}
The necessity to elaborate a theory of nutation and precession matching the accuracy of very modern techniques as Very Long Baseline Interferometry and Lunar Laser Ranging led recently to various works. We discuss here the good agreement between those related to the nutation when considering the Earth as a solid body. In comparison we show the uncertainty concerning the modelisation of the transfer function leading to theoretical determination of the nutation coefficients when including dominant geophysical characteristics.
\end{abstract}

\section{Introduction}

The conventional IAU80 series of nutation of the Earth (Seidelmann, 1982) are based on two fundamental works which are: first, the computation of the coefficients of the nutation when considering the simplified case of a rigid Earth, carried out by Kinoshita (1977). Secondy, the calculation of the nutation of the Earth by taking into account the various geophysical properties of the Earth, as was done by Wahr (1979). Notice, that the second work is directly dependent on the first one. In other words, the amplitude of each coefficient of nutation for a non-rigid Earth model, both in longitude and in obliquity, is derived from its amplitude when considering the Earth as rigid. The ratio between them depends on the frequency of the oscillation itself, and is calculated for each coefficient from a formula involving the frequencies of three fundamental modes of the rotation of the Earth. In the following, we compare recent results related to the nutation for a rigid Earth model presented by Williams (1994, 1995, 1996), Hartmann and Soffel (1996), Souchay and Kinoshita (1996a,b), Roosbeek and Dehant (1996) and Bretagnon et al., (1996). Then, we show the very good agreement between these studies when comparing the differences between the values of the coefficients of nutation given by observational data with those established analytically for a non-rigid Earth model.

I. M. Wytrzyszczak, J. H. Lieske and R. A. Feldman (eds.),

Dynamics and Astrometry of Natural and Artificial Celestial Bodies, 319, 1997.

(C) 1997 Kluwer Academic Publishers. Printed in the Netherlands. 


\section{Rigid Earth Nutation: Present State}

\subsection{SUMMARY OF RECENT RESULTS ON RIGID EARTH NUTATIONS}

It appears convenient to summarize each study:

- Williams (1994) made new important remarks with respect to previous works. Firstly, he calculated the Earth's obliquity rate with respect to the plane of the ecliptic of a given epoch, which was already pointed out by Woolard (1953), but not mentioned in the various papers afterwards. His estimation is: $\dot{\varepsilon}=-0.0254^{\prime \prime} /$ cy. Notice, that this obliquity rate has been confirmed by observational data (Souchay et al., 1995). Secondly, he showed that effects due to the tilted lunar mean plane lead to in-phase and out-of-phase components of nutation for the terms of argument $\Omega$ and $2 \Omega$. Moreover, Williams computed time and obliquity dependence of precession and obliquity rates which are needed to calculate the evolution of precession and obliquity with time, and he gave an estimation of the dynamical ellipticity of the Earth $\left(H_{D}=0.0032737634\right)$ in a similar way as in Kinoshita and Souchay (1990) but based on recent estimations of the general precession in longitude. In another paper, Williams (1995) calculated the direct effect of the planets with a truncation at the level of $1 \mu$ as instead of $5 \mu$ as in the series of Kinoshita and Souchay (1990).

- Hartmann and Soffel (1996) calculated series of rigid Earth nutation which contrast significantly with the traditional method of computation. The coefficients are calculated indirectly by the intermediary of new and highly accurate tidal potential development (Hartmann and Wenzel, 1995). Their computations include all various effects already investigated by Kinoshita and Souchay (1990), but at the level of $0.45 \mu$ as instead of $5 \mu$ as as in this last work. Moreover, they showed that the $J_{4}$ geopotential gives birth to coefficients for the $18.6 \mathrm{yr}$ component at the level of a few $\mu$ as. In this paper also, a value of the dynamical ellipticity is given $\left(H_{D}=0.0032737925\right)$.

- Roosbeek and Dehant (1996) calculated the nutation for a rigid Earth model in a classical way, by integrating the equations relative to the angular momentum. For this purpose, they used the same ephemerides as Kinoshita and Souchay (1990), but in their new version [VSOP88 (Bretagnon and Francou, 1988)] to calculate the potential exerted by the Sun and the planets, and ELP2000 (Chapront-Touzé and Chapront, 1988).

- Souchay and Kinoshita (1996a) re-computed the precession and the main terms (luni-solar influence on the $J_{2}$ geopotential) of the nutation for a rigid Earth model in the same way as during the reconstruction of the theory (Kinoshita and Souchay, 1990) established by Kinoshita (1972, 1977), starting from a formalism based on the Hamiltonian of a rotating body. For this purpose they used the same ephemerides as Roosbeek and Dehant, and took into account the correction of the general precession in 
longitude $p_{A}$ with respect to the conventional value (Lieske et al., 1977), coming from various observations, as was noticed by Williams (1994). They showed that this correction influences directly the value of $H_{d}$ in a substantial way, which means that all coefficients of nutation have to be modified by a same relative amount. In addition to the corrections due to the new value of $p_{A}$, Souchay and Kinoshita noticed some errors in the tables of Kinoshita and Souchay (1990), and some further corrections due to the fact that all the terms with a relative $10^{-11}$ value were kept in the luni-solar potential instead of $10^{-9}$ in this last work. All the coefficients which have been subject to a change larger than $1 \mu$ as for the various reasons explained above were listed in a single recapitulative table. In a second paper, Souchay and Kinoshita (1996b) re-computed the coefficients of rigid Earth nutation due to the second-order geopotential involving the coefficients $J_{3}$, $J_{4}, C_{2,2}$ and $S_{2,2}$, and due to the direct effects of the planets, with the same level of truncation as Williams (1995) of $1 \mu$ as.

- Bretagnon et al., (1996) computed the rigid Earth precession and nutation starting from the equations relative to the angular momentum and the semi-analytical theories of the motion of the Moon, the Sun and the planets of the Bureau des Longitudes. Their work is complete, they took into account the influence of the Sun, the Moon, and all the planets on the potential of the Earth limited to the $4^{\text {th }}$ order. The secular trends for $\psi$ and $\varepsilon$ are listed, as well as the largest term of each contribution.

\subsection{COMPARISONS BETWEEN RIGID EARTH NUTATIONS}

In view of the abundant production of recent works dealing with rigid Earth nutation, as shown in the last paragraph, it is possible to get a clear insight of the state-of-the-art of the agreements and disagreements on this topic, especially by comparing results of the amplitudes of the coefficients obtained with different theories. In the following, we make a review of these comparisons by considering separately each kind of nutation.

- Main terms of nutation

These terms come from the luni-solar gravitational action on the $J_{2}$ potential characterizing the flattening of the Earth. The largest coefficients $(18.6 \mathrm{yr}, 9.3 \mathrm{yr}, 1 \mathrm{yr}, 1 / 2 \mathrm{yr}, 27.5 \mathrm{~d}, 13.66 \mathrm{~d})$ belong to this category. Although their computations are carried out by Souchay and Kinoshita (1996a) on one side, with the help of canonical equations, and by Roosbeek and Dehant (Roosbeek, 1996, private communication) on the other side, starting from the equations relative to the angular-momentum, the discrepancy in amplitude is generally very small. In longitude, it does not exceed $1 \mu$ as for 20 among the 33 largest coefficients. This discrepancy exceeds $50 \mu$ as for only 3 coefficients $(18.6 \mathrm{yr}, 9.3 \mathrm{yr}, 27.5 \mathrm{~d})$. In obliquity, the results are still 
better with a discrepancy not exceeding $1 \mu$ as for 14 terms among the 19 largest ones, and only two terms (18.6 yr, $9.3 \mathrm{yr})$ with a discrepancy larger than $50 \mu$ as. Some results obtained by Williams (1996, private communication) for the 10 largest coefficients give quite similar results with a better agreement (within $20 \mu \mathrm{as}$ ) with Souchay and Kinoshita (1996a) for the $18.6 \mathrm{yr}$ and $9.3 \mathrm{yr}$ components, both in longitude and obliquity. Hartmann and Soffel (1996), when computing the nutation from tidal series, found significantly bigger differences with Souchay and Kinoshita (1996a), reaching $25 \mu$ as for 30 coefficients in $\Delta \psi$ and $10 \mu$ as for 28 coefficients in $\Delta \varepsilon$. Notice, that a large majority of these differences concern long periodic terms, and that these differences are excessively big for the terms of periods close to $18.6 \mathrm{yr}$ ( 5.355 mas for the $6798.383 \mathrm{~d}$ in-phase coefficient and 1.953 mas for the $6786.317 \mathrm{~d}$ in-phase coefficient) and at the level of a few tenths of mas for several other ones. Nevertheless, Hartmann and Soffel clearly state that for these terms their method of calculation is not well-suited, so that they should not be taken seriously into account and no real contradiction exists. Bretagnon et al. (1996), in conclusion of their computation of rigid Earth nutation, indicate that their solution agrees well with the secular developments of Williams (1994) and the periodic series of Souchay and Kinoshita (1996). However, they mentioned a $0.250 \mu$ as difference for the out-of-phase component in the term of period $18.6 \mathrm{yr}$, which remains unexplained.

- Indirect planetary effects

They are due to the perturbation caused by the planets on the luni-solar potential. They have been computed by Souchay and Kinoshita (1996a) up to $1 \mu$ as. Souchay (1990). A few changes and corrections have been noticed in the tables of this last paper, confirming exactly the remarks given by Williams (1995, private communication). They have been listed by Souchay and Kinoshita (1996a).

- Effect of the $J_{3}$ and $J_{4}$ geopotential.

The influence of $J_{3}$ is characterized by 22 terms up to $0.5 \mu \mathrm{as}$, with various periods, as was shown by Souchay and Kinoshita (1996b), who found a remarkable agreement with the values calculated by Hartmann et al., (1996), starting from the tidal potential. The difference in amplitude does not exceed a few tenths of $\mu$ as. The influence of $J_{4}$ is significant only for the two terms of periods $9.3 \mathrm{yr}$ and $18.6 \mathrm{yr}$, as demonstrated by these authors. We have, in microarcseconds: $\Delta \psi_{J 4}=0.73 \sin \Omega-0.61 \sin 2 \Omega$, $\Delta \varepsilon_{J 4}=6.83 \cos \Omega-0.61 \cos 2 \Omega$.

- Effect of the triaxiality of the Earth.

The Earth is not a perfect axisymetric body. The influence of the Moon and the Sun on its triaxialty characterized by the coefficients $C_{2,2}$ and $S_{2,2}$ of the geopotential gives quasi semi-diurnal nutation terms, as calculated by Kinoshita and Souchay (1990), and more precisely by Hartmann and 
Soffel (1996) as well as by Souchay and Kinoshita (1996b). The comparison between the values of the coefficients in these two last works is also quasiperfect, exceeding $1 \mu$ as only for one of them.

- Direct action of the planets.

Williams (1995) and Souchay and Kinoshita (1996b) calculated the coefficients of rigid Earth nutation coming from this action by quite different methods, as explained before. Nevertheless, the agreement is quite remarkable between the two works, the difference of amplitude for each coefficient retained not exceeding $0.1 \mu$ as except for 2 from among the 158 coefficients listed, up to $0.5 \mu \mathrm{as}$, both in longitude and obliquity. Notice, that Kinoshita and Souchay (1990) obtained only 35 coefficients up to $5 \mu$ as.

\section{Influence of the Rigid Earth Nutation Model on the Non-Rigid Earth Nutation Model}

To estimate the influence of the model of rigid Earth nutation on the nonrigid Earth nutation, a straightforward procedure consists in applying the same analytical transformations due to the geophysical properties, to two different models of rigid Earth nutation, and to compare the coefficients after transformation. This was done by Souchay et al., (1995) who applied the analytical formula given by Wahr (1979) to new series of nutation for a rigid Earth model (Kinoshita and Souchay, 1990), instead of the old ones (Kinoshita, 1977) as it was the case for the establishment of the conventional IAU80 series of nutation. The study shows that for the leading terms of nutation ( $18.6 \mathrm{yr}, 1 \mathrm{yr}, 1 / 2 \mathrm{yr}, 13.66 \mathrm{~d})$ the differences are very small in comparison with the difference between the conventional value and the value determined from VLBI observations. This means that the influence of the rigid Earth model value of these terms is not significant with respect to the imperfections in the modeling of the Earth's interior response. Souchay et al., (1996) showed that after fitting eight of the nine largest nutation terms that are affected with geophysical modeling, and adopting an empirical Free Core Nutation of period $433 \mathrm{~d}$, the VLBI results were able to distinguish between the old and the new series for terms which are not expected to be modified by changes in geophysical modeling. This was in a great part due to the fact that the old rigid Earth nutation series (Kinoshita, 1977) were truncated to 0.1 mas. Coefficients were limited to this value, and all the coefficients smaller than this value were not included. On the contrary, the series of Kinoshita and Souchay (1990) include all the coefficients up to 0.005 mas. Notice, that the uncertainty of the determination of the 42 largest coefficients not fitted as above, to VLBI data, is ranging between \pm 0.020 mas and \pm 0.030 mas (Souchay et al., 1996), whereas the difference between the theoretical values of these coefficients for a rigid Earth model 
as given by Souchay and Kinoshita (1996a,b) and Roosbeek and Dehant, generally does not exceed 0.001 mas $(1 \mu$ as). For the $18.6 \mathrm{yr}$ term the difference between VLBI estimation and the IAU conventional value is -7.53 mas in longitude and 1.49 mas in obliquity (Souchay et al., 1995), which is far larger than the difference between two theoretical values for a rigid Earth model, which is of about 0.02 mas as seen before.

\section{Conclusion}

In view of the precedent paragraphs, we assume that the agreement between rigid Earth nutation theories (Souchay and Kinoshita, 1996a,b; Roosbeek and Dehant, 1996; Williams, 1994, 1995; Bretagnon et al., 1996: Hartmann and Soffel, 1996) is quite satisfactory in comparison with the uncertainty of non-rigid Earth modeling and of VLBI individual estimations. In some parts of the rigid Earth nutation $\left(J_{3}, J_{4}\right.$, triaxiality, direct planetary effects) we can even consider that a perfect agreement achieved for the values given by quite different theories does not generally exceed $1 \mu$ as.

\section{References}

Bretagnon, P., Rocher, P., and Simon, J.L.: 1996, Astron. Astrophys., submitted.

Bretagnon, P. and Francou, G.: 1988, Astron. Astrophys. 202, 309.

Chapront-Touzé, M. and Chapront, J.: 1988, Astron. Astrophys. 190, 342.

Hartmann, T. and Wenzel, H.G.: 1995, Geophys. Res. Lett. 22, 3553.

Hartmann, T., Williams, J.W. and Soffel, M.: 1996, Astron. J. 111, 1400.

Hartmann, T. and Soffel, M.: 1996, Celest. Mech., in press.

Kinoshita, H.: 1972, Publ. Astron. Soc. Jpn. 24, 423.

Kinoshita, H.: 1977, Celest. Mech. 26, 296.

Kinoshita, H. and Souchay, J.: 1990, Celest. Mech. 48, 187.

Lieske, J.H., Lederle, T., Fricke, W. and Morando, B.: 1977, Astron. Astrophys. 58, 1.

Souchay, J. and Kinoshita, H.: 1996, Astron. Astrophys. 190, 312, 1017.

Souchay, J. and Kinoshita, H.: 1996, Astron. Astrophys., in press.

Souchay, J., Feissel, M., Bizouard, C., Capitaine, N. and Bougeard, M.: 1995, Astron. Astrophys. 299, 277.

Souchay, J., Feissel, M. and Ma, C.: 1996, Astron. Astrophys., Suppl. Ser. 116, 473.

Roosbeek, F. and Dehant, V.: 1996, Celest. Mech., in press.

Seidelmann, P.K.: 1982, Celest. Mech. 27, 79.

Wahr, J.M.: 1979, Ph.D. Thesis, University of Colorado, Boulder, Colorado.

Williams, J.G.: 1994, Astron. J. 108, 711.

Williams, J.G.: 1995, Astron. J. 110, 1420.

Woolard, E.W.: 1953, Astron. Papers for the American Ephemeris and Nautical Almanac $X V 1$, U.S. Gov. Print. Office, Washington. 